\title{
Healthcare Payer
}

National Cancer Institute

\section{Source}

National Cancer Institute. Healthcare Payer. NCI Thesaurus. Code C70686.

An entity, org anization, government, corporation, health plan sponsor, or any other

financial agent who pays a healthcare provider for the healthcare service rendered to a patient or reimburses the cost of the healthcare service. 